\title{
Data Association in Communication-Constrained Environments Based on Discrete Fréchet Distance
}

\author{
Huan Shao ${ }^{+}$and Yue Wang \\ Department of Electronic Engineering, Tsinghua University, Beijing, China
}

\begin{abstract}
The performance of traditional methods in data association degrades significantly in communication-constrained environments. This paper presents a data association algorithm based on discrete Fréchet distance that establishes metrics on the shape of a target track. It is shown to have good resolving power of distinguishing objects with less data required, which is then verified by simulation results.
\end{abstract}

Keywords: data association, discrete Fréchet distance, metrics, communication-constrained, identification friend or foe.

\section{Introduction}

Target data association is one of the key procedures of sensor data fusion. The purpose of traditional data association is to determine the correlation of multiple-site track data measured by multiple sensors, in order to help fusion system efficiently estimate target states. Besides, data association also has other applications, such as identification friend or foe (IFF), where the working conditions are quite different from those in traditional target state estimations.

Fig. 1 shows a work scene where data association is used to identify friend or foe. Radar $\mathrm{S}_{1}$ observes some objects in the surveillance region. However, It is unknown whether these objects are friends or not. So the operator asks friend targets for their track data. Object $A$ is one of the objects observed by radar $S_{1}$. When a request is received, those friend targets, around object $\mathrm{A}$ within a certain range, return data as required. Let data set $D_{1}$ be the measurements of object $\mathrm{A}$, and $D_{2}$ be the data returned from friend targets. Object A will be judged as a friend target, if a target in $D_{2}$ can be found matching data in $D_{1}$. Otherwise, object A would be judged as not a friend. Therefore, target IFF is completed. Here, we can find that IFF can be formalized as a data association problem: whether a correspondence can be built between data set $D_{1}$ and $D_{2}$.

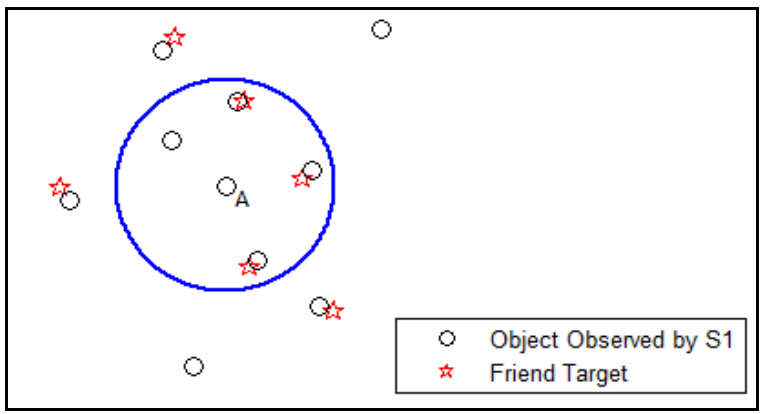

Fig. 1: A work scene of IFF

In these cases, communication bandwidth is extremely limited. In most cases, data are transferred

Corresponding author. Tel.: + 86-021-62796180; fax: +86-021-62796180.

E-mail address: shaoh14@mails.tsinghua.edu.cn. 
through convert channels, where each data stream is strictly limited to no more than 120 bytes. As a result, the transmitted data of targets have to either be partial or contain significant quantization errors. While traditional association algorithms are used to estimate states of targets, which needs all the states to be transmitted. This leads to the difference between traditional data association and the association for IFF.

Most of the existing association algorithms, such as the basic correlation-gate-based algorithm[1], the mainstream probability-based algorithms[2-4], and their optimized algorithms[5,6], are all designed to improve the correlation accuracy without considering the communication-constrained scenarios.

This paper presents a data association method using discrete Fréchet distance (DFD) to improve the performance of IFF target association with lower volume of data. We first illustrate its advantages over traditional algorithms from the perspective of metrics, which are then verified by a Monte Carlo simulation for a representative application example.

The outline of this paper is as follows. In section 2, we formulate the association problem in communication-constrained environments. In section 3, we introduce our data association method. Section 4 presents the simulation platform and results. Finally, Section 5 concludes our work.

\section{Problem Formulation}

In this paper, the default inputs of data association system only contain targets' positions and time.

Suppose that data set $D_{1}$ (measurements of radar $\mathrm{S}_{1}$ ) and $D_{2}$ (data returned from friend targets, also called cooperative targets) contain data of various targets in a certain range in 2D space. They both have systematic errors (biases) and random errors (noise). The errors consist of azimuth errors and range offset errors. Let $\left(b_{r}^{D_{j}}, b_{\theta}^{D_{j}}\right)$ be the sensor bias of data set $D_{j},\left(n_{r}^{D_{j}}, n_{\theta}^{D_{j}}\right)$ be the noise of $D_{j}$, where $j=1,2$. The biased measurements of target $i$ detected by the sensor in $D_{j}$ at time $\mathrm{k}$ are

$$
\left\{\begin{array}{l}
r_{i}^{D_{j}}(k)=\hat{r}_{i}^{D_{j}}(k)+b_{r}^{D_{j}}+n_{r}^{D_{j}} \\
\theta_{i}^{D_{j}}(k)=\hat{\theta}_{i}^{D_{j}}(k)+b_{\theta}^{D_{j}}+n_{\theta}^{D_{j}}
\end{array}\right.
$$

where $\left(\hat{r}_{i}^{D_{j}}(k), \hat{\theta}_{i}^{D_{j}}(k)\right)$ is the real position of target $i$ from the sensor in $D_{j}$ at time k.

The position coordinates of target $i$ detected by the sensor in $D_{j}$ at time $\mathrm{k}$ are

$$
\left\{\begin{array}{l}
x_{i}^{D_{j}}(k)=x_{D_{j}}+r_{i}^{D_{j}}(k) \sin \theta_{i}^{D_{j}}(k) \\
y_{i}^{D_{j}}(k)=y_{D_{j}}+r_{i}^{D_{j}}(k) \cos \theta_{i}^{D_{j}}(k)
\end{array}\right.
$$

where $\left(x_{D_{j}}, y_{D_{j}}\right)$ are the coordinates of the sensor in $D_{j}$.

A track point refers to the values of all the track coordinates at a given time.

Because of the sensor biases and noise, measurements by different sensors are not the same as real tracks of targets $l_{i}=\left\{\left(x_{i}(k), y_{i}(k)\right\}\right.$, and they are also different from each other. Furthermore, since $D_{2}$ refers to the data returned from cooperative targets and $D_{1}$ may contain the data of a friend target or other target, the target groups of the two data sets are different. In addition, communication bandwidth is limited while cooperative targets need to return data according to the request, which means the data of association is limited.

In summary, the abstract problem is to associate one target in $D_{1}$ with all the targets in $D_{2}$ while the upper bound of data volume is given.

\section{Association Method}

Data association can be divided into two steps. First, establish metrics to describe relations among target tracks. Second, introduce a criterion to deduce the correlation. As a result, there are two aspects to improve the performance, i.e., the accuracy of data association. One is the metrics aspect. This means optimizing metrics, improving the resolving power of metrics, and therefore increasing the contrast of different correlations. The other is the system aspect. That is to change judgement tactics and to choose effective criteria according to prior knowledge and application scenarios. This paper focuses on the metrics aspect. 
There are two types of common metric methods when the inputs of the data association system only contain targets' positions and time. One is the typical way that uses the feature of single pair of track points, such as the distance between two track points at one time. Based on this metrics, data association can be done by using correlation gates[1] or probability-based[2] criteria. The other type establishes metrics based on integrated features of target tracks, such as velocities and directions of tracks. Such methods commonly serve as compensation to the former type. Similarly, they can work with any criterion.

Nearest neighbor algorithm(NN)[1] and probabilistic data association(PDA)[2] are two common algorithms in traditional data association. They both use statistic-based distances among track points as metrics, but their criteria are different. NN employs a correlation gate. To the contrary, PDA uses a probabilistic score, which measures the goodness of fit of the hit-to-track association, for target associating and tracking. NN works well only with high SNR and low target density. Its performance degrades significantly when data quality decreases. As for PDA, its effectiveness is severely restricted due to the high computational complexity.

This paper proposes a new metric method that establishes metrics on the shape of a target track. We use discrete Fréchet distance (DFD) as a measure of similarity between two tracks, and then, implement data association based on the similarity between the local and returned tracks. Fréchet distance is widely used in computer vision and pattern recognition[7,8]. It is symmetric and maintains the triangle inequality. Moreover, it is invariant under orientation-preserving reparametrizations[9] As a result, we can leverage Fréchet distance as the metric. As far as we know, this is the first work to introduce this kind of metric method in data association. Compared with traditional metric methods, DFD achieves almost the same resolving power with even less data. While complicated criteria can be applied, in order to show the advantages of this metrics in communication-constrained environments, we choose a sample criterion: correlation gates, to implement data association when comparing the performance.

\subsection{Discrete Fréchet Distance}

Fréchet distance was first defined by Fréchet[9]. It can be used as a measure for the resemblance of curves in a metric space. Thomas and Heikki gave a discrete variation of Fréchet distance for polygonal curves[10].

The tracks of target $i$ and $j: l_{i}(k)(k=0,1, \ldots, n), l_{j}(t)(t=0,1, \ldots, m)$ are modeled as polygonal curves $P$ : $[0, n] \rightarrow V$ and $Q:[0, m] \rightarrow V$, where $(V, d)$ is an Euclidean space and $n, m$ are positive integers. Then the track points can be regarded as the end points of the line segments of the polygonal curves: $\sigma(P):(P(0), P(1), \ldots P(n)), \sigma(Q):(Q(0), Q(1), \ldots Q(m))$. Hence, the similarity between two tracks can be described by the discrete Fréchet distance between the two polygonal curves. It takes into account the location and ordering of the points along the curves.

Let $\sigma(P)=\left(u_{1}, \ldots, u_{p}\right), \sigma(Q)=\left(v_{1}, \ldots, v_{q}\right)$ be the corresponding sequences. A coupling $L$ between $P$ and $Q$ is a sequence $\left(u_{a_{1}}, v_{b_{1}}\right),\left(u_{a_{2}}, v_{b_{2}}\right), \ldots,\left(u_{a_{w}}, v_{b_{w}}\right)$ of distinct pairs from $\sigma(P) \times \sigma(Q)$ such that $a_{1}=1$, $b_{1}=1, a_{w}=p, b_{w}=q$, and for all $i=1, \ldots, w$, we have $a_{i+1}=a_{i}$ or $a_{i+1}=a_{i}+1$, and $b_{i+1}=b_{i}$ or $b_{i+1}=b_{i}+1$. Thus, a coupling has to respect the order of the points in $P$ and $Q$. The length of the couple $L$ is the length of the longest link in $L$, that is,

$$
\|L\|=\max _{i=1, \ldots, w} d\left(u_{a_{i}}, v_{b_{i}}\right)
$$

Given polygonal curves $P$ and $Q$, their discrete Fréchet distance is defined to be

$$
\delta_{d F}(P, Q)=\min \{\|L\| \mid L \text { is a coupling between } P \text { and } Q\}
$$

In reality, the measurements of radars introduce systematic and random errors. Therefore, the locations of the points along the curves are imprecise. We can handle these cases by an optimized algorithm ${ }^{[11]}$.

The shorter the discrete Fréchet distance is, the more similar two polygonal curves are, which means it is more likely that the two target tracks, represented by curves, originate from the same target.

According to the correlation-gate criterion, given a correlation gate $\varepsilon$, if we have $\delta_{d F}(P, Q)<\varepsilon$, then we 
claim the two curves are similar, i.e., the two tracks originate from the same target. Otherwise, they don't.

\subsection{Comparing the Resolving Power of Different Metrics}

In the association system, when effective data become less, the performance of data association gets worse. From the perspective of metrics, that means the resolving power of metrics degrades. It may lead to two consequences. One is that tracks of two different targets become similar and hence hard to distinguish. The other is that two tracks of the same target become more different.

This paper compares discrete Fréchet distance as the metric method in data association with conventional metrics: statistical distance, which refers to the arithmetic average of distances between two track points at one time. According to section 3.1, DFD shares the same property with statistical distance: the smaller the value, the closer the two tracks, the more likely that they originate from the same target.

We use these two metric methods to describe relations among target tracks respectively in the same association scenario described in the following section.

The distribution ranges of the two distances are shown in Fig. 2 where real lines indicate the distribution range of the distance in the situation that the unknown object is a friend target and dotted lines show the distribution range when the unknown object is not a friend. Fig. 2 shows how the resolving power of the two metric methods changes with different numbers of track points. It demonstrates that both methods distinguish objects better with more data. Furthermore, DFD outperforms statistical distance. DFD eliminates the confusion among targets with only a few (e.g. 10) track points, while statistical distance does not.
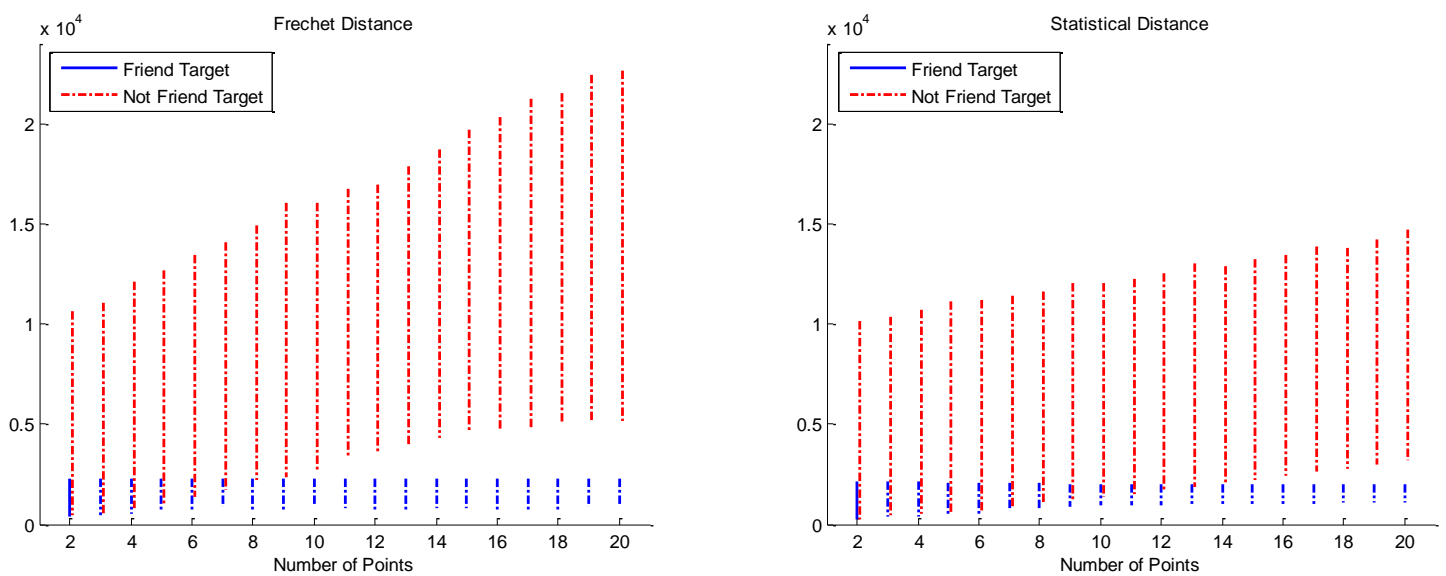

Fig. 2: Distribution ranges of the two distances with different numbers of track points

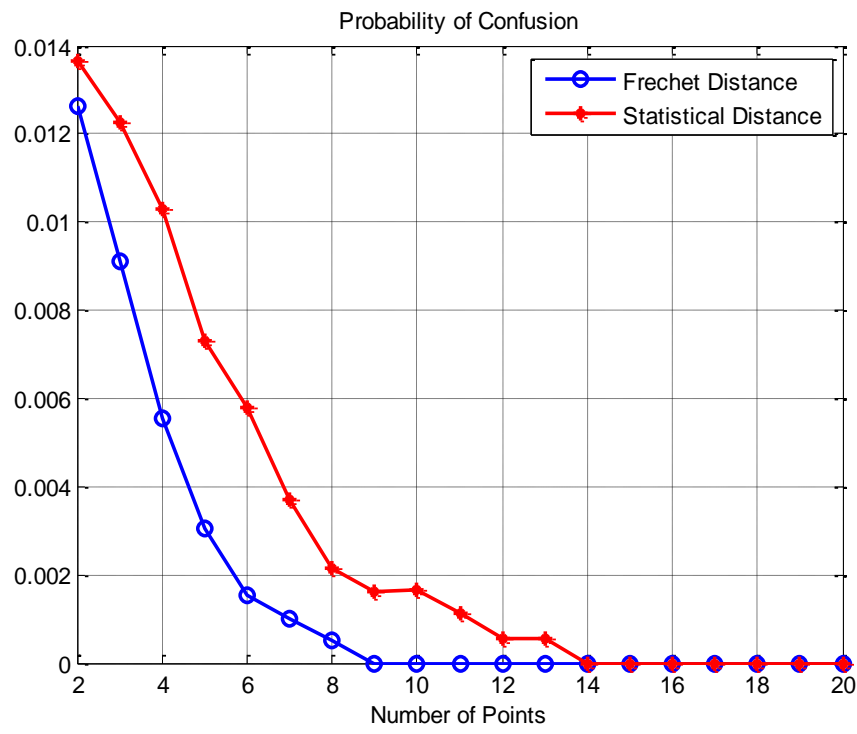

Fig. 3: Probability of confusion of the two distances with different numbers of track points 
Fig. 3 shows the probability of confusion of the two distances with different numbers of track points. In the same association scenario, DFD makes less confusion than statistical distance. As the number of track points increases, the probability of confusion made by DFD decreases rapidly, outperforming its counterpart.

Therefore, it is confirmed that discrete Fréchet distance has stronger resolving power than statistical distance in communication-constrained environments, i.e., it distinguishes objects better.

\section{Simulation}

\subsection{Simulation Platform}

Simulation scenarios are randomly generated according to the parameters of the simulation system. The parameters include the number of targets, the number of track points of each target, the sampling rate, the proportionality of friend targets, the upper bound of target velocity and the minimum turning radius of targets. To simulate reality, the tracks of targets are composed of irregular arcs and lines. The generated targets are relatively concentrated so that the actual performance of association algorithms can be evaluated clearly.

We extract the simulation parameters from the data sheets of warships. The upper bound of velocity is $16 \mathrm{~m} / \mathrm{s}$ and the minimum turning radius is $800 \mathrm{~m}$. Fig. 4 shows an association scenario. There are 20 targets, of which 15 are friend targets. Each target has 20000 points, sampled at 4 points per second.

We simulate two radars $\mathrm{S}_{1}$ and $\mathrm{S}_{2}$, which collect measurements regarded as data set $D_{1}$ and $D_{2} . \mathrm{S}_{1}$ and $\mathrm{S}_{2}$ are located in $(15 \mathrm{~km}, 20 \mathrm{~km})$ and $(-5 \mathrm{~km},-5 \mathrm{~km})$ respectively. For each radar, the scanning period is $5 \mathrm{~s}$; the detection coverage is a circle with radius $100 \mathrm{~km}$; the bias in range is $250 \mathrm{~m}$; the bias in azimuth is $2.5^{\circ}$. The random measurement noise is white Gaussian with a radial standard deviation of $25 \mathrm{~m}$ and a tangential standard deviation of $0.25^{\circ}$. Since what $S_{2}$ observes represents the information sent back from friend targets, the observed target groups of the two radars are different.

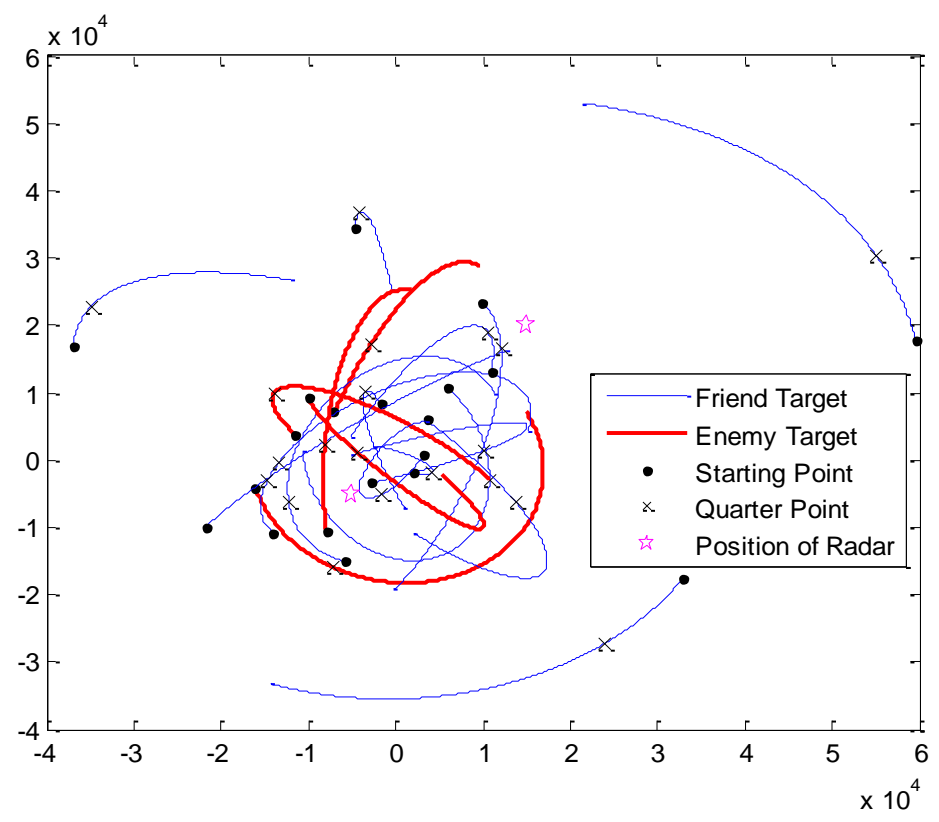

Fig. 4: Simulation platform

We traverse each of the targets in the scenario as the unknown target for $S_{1}$. Then we sweep the inquiry time with a fixed increment and generate $20 * 80$ experiments. Each experiment represents a combination of one inquiry, one response and one data association analysis. The total 1600 experiments are leveraged to evaluate the association accuracy of different algorithms. All results are based on 50 Monte Carlo runs.

\subsection{Simulation Results}

\section{A. Performance Comparison}

We compare the resolving power of DFD and conventional metrics in section 3.2. This section compares the performance of association algorithms based on different metric methods. To show the performance 
improvement gained from the metrics aspect, we choose a sample criterion: correlation gates. Since NN employs a correlation gate based on statistical distance, it is fit for comparison.

Fig. 5 shows that as the data volume increases, the association accuracy of both the two methods increases. Our algorithm based on DFD performs better than NN robustly. More importantly, our algorithm requires less data than $\mathrm{NN}$ when achieving similar accuracy.

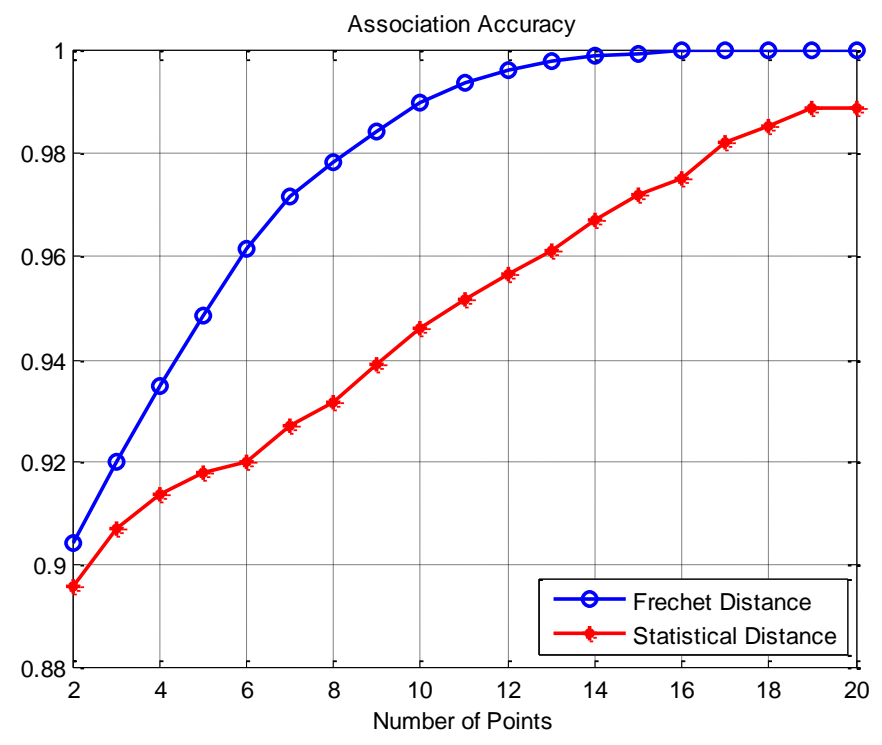

Fig. 5: Association accuracy of the two methods with different numbers of track points

\section{B. Sensitivity Analysis}

We increase the number of targets in the simulation scenario from 20 to 30.

Fig. 6 shows the effect of target density on metrics distinguishing objects. When the density of targets increases, for both the two distances, the probability of confusion increases, while DFD makes less confusion than statistical distance. Furthermore, as the number of track points increases, DFD's probability of confusion drops at a much faster speed, i.e., its resolving power improves more quickly.

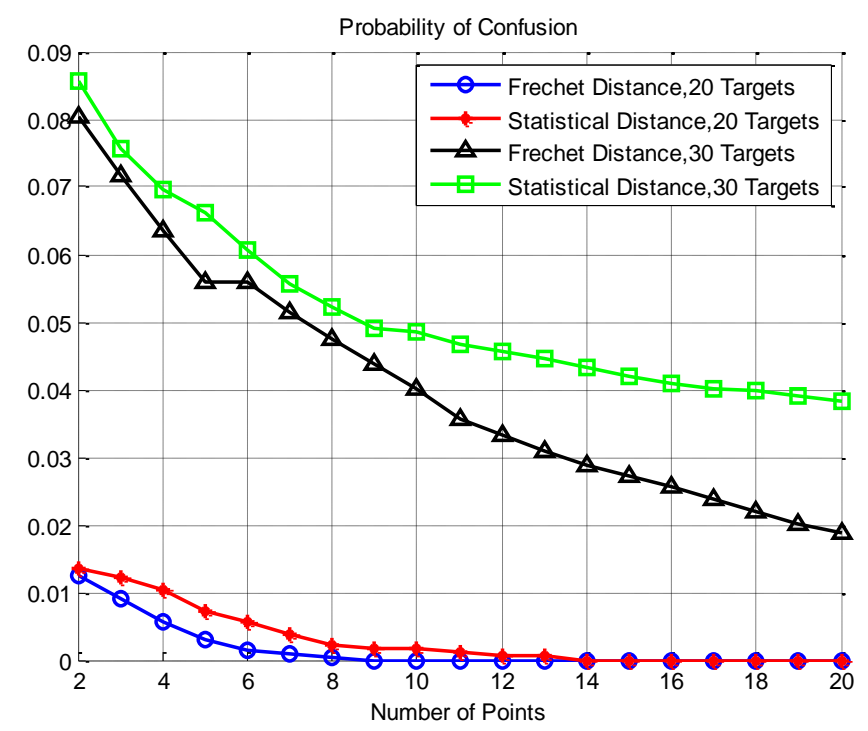

Fig. 6: Probability of confusion of the two distances in different target density with different numbers of track points

Fig. 7 shows the effect of biases and noise on metrics distinguishing objects. DFD is more robust under biases and noise than statistical distance. Fig. 7 also shows that DFD is not sensitive to range offset errors, but vulnerable to azimuth errors. Its resolving power decreases when tangential biases or noise increase.

In general, the simulation results show that DFD performs better than conventional metrics for data 
association in communication-constrained environments with different target density, biases and noise.
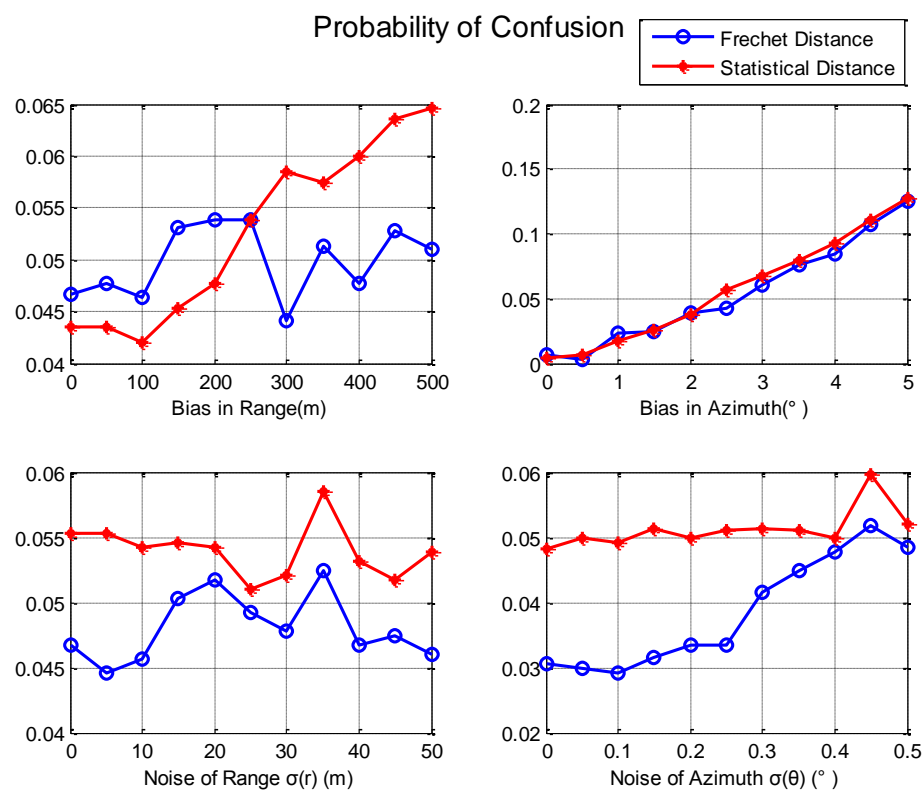

Fig. 7: Probability of confusion of the two distances in different biases and noise with different numbers of track points

\section{Conclusion}

This paper presented a data association algorithm based on discrete Fréchet distance and illustrated its advantages over traditional methods through both theoretical analysis and simulation verification from the perspective of metrics: it substantially relaxes the required data volume of data association. As a result, it is fit for IFF data association, especially in communication-constrained environments. It achieves comparable or even better accuracy than its conventional counterparts with less data required.

\section{References}

[1] Kanyuck, Allen, and Robert A. Singer. "Correlation of multiple-site track data." Aerospace and Electronic Systems, IEEE Transactions on 2 (1970): 180-187.

[2] Bar-Shalom Y, Tse E. Tracking in a cluttered environment with probabilistic data association[J]. Automatica, 1975, 11(5): 451-460.

[3] Rasmussen C, Hager G D. Probabilistic data association methods for tracking complex visual objects[J]. IEEE Transactions on Pattern Analysis and Machine Intelligence, 2001, 23(6): 560-576.

[4] Musicki D, Evans R. Joint integrated probabilistic data association: JIPDA[J]. IEEE transactions on Aerospace and Electronic Systems, 2004, 40(3): 1093-1099.

[5] Roecker J A, Phillis G L. Suboptimal joint probabilistic data association[J]. IEEE Transactions on Aerospace and Electronic Systems, 1993, 29(2): 510-517.

[6] Bar-Shalom Y, Daum F, Huang J. The probabilistic data association filter[J]. IEEE Control Systems, 2009, 29(6).

[7] Godau M. On the complexity of measuring the similarity between geometric objects in higher dimensions[D]. Freie Universität Berlin, 1999.

[8] Benedikt L, Kajic V, Cosker D, et al. Facial Dynamics in Biometric Identification[C]//BMVC. 2008: 1-10.

[9] Fréchet M M. Sur quelques points du calcul fonctionnel[J]. Rendiconti del Circolo Matematico di Palermo (1884-1940), 1906, 22(1): 1-72.

[10] Eiter T, Mannila H. Computing discrete Fréchet distance[R]. Tech. Report CD-TR 94/64, Information Systems Department, Technical University of Vienna, 1994.

[11] Ahn H K, Knauer C, Scherfenberg M, et al. Computing the discrete Fréchet distance with imprecise input[J]. International Journal of Computational Geometry \& Applications, 2012, 22(01): 27-44. 